\title{
Two Dipeptide-Bound Pyrralines with Ile or Ala: A Study on Their Synthesis, Transport across Caco-2 Cell Monolayers and Interaction with Aminopeptidase $\mathbf{N}$
}

\author{
Haiping $\mathrm{Qi}^{1}$, Zhenhui Zhang ${ }^{1}$, Xia Zhang ${ }^{1}$, Bing $\mathrm{Li}^{1 *}$, Lin $\mathrm{Li}^{1,2^{*}}$ \\ 1 School of Food Science and Engineering, Guangdong Province Key Laboratory for \\ Green Processing of Natural Products and Product Safety, Ministry of Education \\ Engineering Research Center of Starch \& Protein Processing, South China University \\ of Technology, Guangzhou, 510640, China
}

2 School of Chemical Engineering and Energy Technology, Dongguan University of Technology, College Road 1, Dongguan, 523808, China

\section{Materials and Methods}

\section{Synthesis and Purification of Pyrr-Ile and Pyrr-Ala}

Boc-TBS-Pyrr-Ile (Ala) synthesis: TBS-protected dihydropyranone (39 mg, 0.15 $\mathrm{mmol}$ ) and Boc-Lys-Ile (83 mg, $0.23 \mathrm{mmol})$ or Boc-Lys-Ala (73 mg, $0.23 \mathrm{mmol}$ ) were dissolved in $1 \mathrm{~mL}$ THF- $\mathrm{H}_{2} \mathrm{O}(1: 1)$ solution, and then the suspension was heated at $40{ }^{\circ} \mathrm{C}$ for $7.5 \mathrm{~h}$ with stirring constantly. The reaction mixture was extracted three times with ethyl acetate (2 mL per time) after dilution with $\mathrm{H}_{2} \mathrm{O}(2 \mathrm{~mL})$. The collected organic layer washed with $\mathrm{H}_{2} \mathrm{O}(2 \mathrm{~mL})$ and saturated $\mathrm{NaCl}(2 \mathrm{~mL})$ in turn. Dried organic layer over anhydrous $\mathrm{MgSO}_{4}$, it helps to remove water. The filtered organic layer was dried under reduced pressure to obtain crude Boc-TBS-Pyrr-Ile and Boc-TBS-Pyrr-Ala. Then, 
silica gel (ethyl acetate/hexanes, 1. 25:75, 2. 50:50, 3. 75:25, v/v) was used for purification to attain pure Boc-TBS-Pyrr-Ile (1) and Boc-TBS-Pyrr-Ala (2), yellowbrownish solids.

Boc-Pyrr-Ile (Ala) synthesis: compound 1 (37 mg, $0.06 \mathrm{mmol}$ ) or compound 2 (34 $\mathrm{mg}, 0.06 \mathrm{mmol}$ ) was dissolved in $1 \mathrm{~mL} \mathrm{HCl}$ solution (1\%) prepared with $\mathrm{THF}-\mathrm{H}_{2} \mathrm{O}$ $(9: 1)$, then the solution was stirred at room temperature for $7.5 \mathrm{~h}$. After ethyl acetate extraction, saturated $\mathrm{NaCl}$ washing and anhydrous $\mathrm{MgSO}_{4}$ drying, the filtered organic layer was dried under reduced pressure to obtain crude Boc-Pyrr-Ile and Boc-Pyrr-Ala. Purification of silica gel (MeOH/DCM, 1. 5:95, 2. 10:90, 3. 20:80, v/v) afforded pure Boc-Pyrr-Ile and Boc-Pyrr-Ala, yellow solids.

Pyrr-Ile (Ala) synthesis: Boc-Pyrr-Ile (140 mg, $0.3 \mathrm{mmol}$ / Boc-Pyrr-Ala (128 mg, $0.3 \mathrm{mmol})$ was dissolved in TFA- $\mathrm{CH}_{2} \mathrm{Cl}_{2}$ solution $(1: 3,3 \mathrm{~mL})^{1}$, then the solution was stirred at room temperature for $1.0 \mathrm{~h}$. After ethyl acetate extraction, saturated $\mathrm{NaCl}$ washing and anhydrous $\mathrm{MgSO}_{4}$ drying, the filtered organic layer was dried under reduced pressure to obtain crude Pyrr-Ile (3) and Pyrr-Ala (4). Column chromatography $(\mathrm{MeOH} / \mathrm{DCM}, 1.10: 90,2.20: 80,3.30: 70, \mathrm{v} / \mathrm{v})$ was used for purification to afford pure Pyrr-Ile (3), a pale-yellow solid. Pure Pyrr-Ala (4), a pale-yellow solid, was obtained from purification of $\mathrm{C} 18$ column chromatography $\left(\mathrm{MeOH} / \mathrm{H}_{2} \mathrm{O}, 1\right.$. 10:90, 2. 20:80, 3 . 30:70, 4. 40:60, v/v).

\section{Results and Discussion}

BOC-TBS-Pyrr-Ile-(1): HRMS $\left(\mathrm{ESI}^{+}\right)$found $[\mathrm{M}+\mathrm{Na}]^{+}$604.3410, $\mathrm{C}_{29} \mathrm{H}_{51} \mathrm{~N}_{3} \mathrm{O}_{7} \mathrm{SiNa}$ requires $\mathrm{M}^{+}$604.3388. ${ }^{1} \mathrm{H}$ NMR $(600 \mathrm{MHz}$, Methanol-d4) $\delta 9.32(\mathrm{~s}, 1 \mathrm{H}), 6.87(\mathrm{~d}, J=$ 
$4.0 \mathrm{~Hz}, 1 \mathrm{H}), 6.11(\mathrm{~d}, J=4.0 \mathrm{~Hz}, 1 \mathrm{H}), 5.25(\mathrm{~m}, 1 \mathrm{H}), 4.65(\mathrm{~s}, 1 \mathrm{H}), 4.32-4.18(\mathrm{~m}, 2 \mathrm{H})$, 2.12-2.07 (m, 1H), $1.93(\mathrm{~m}, 2 \mathrm{H}), 1.68(\mathrm{~m}, 2 \mathrm{H}), 1.51(\mathrm{~m}, 2 \mathrm{H}), 1.35(\mathrm{~s}, 9 \mathrm{H}), 1.30-1.11$ (m, 5H), 0.88-0.76 (m, 12H), $0.00(\mathrm{~s}, 6 \mathrm{H})$ (Figure S1). IR (neat) v 3364, 2926, 2854, $1704,1656,1548,1459,1382,1271,1164,840,777 \mathrm{~cm}^{-1}$.

Pyrr-Ile-(3): HRMS (ESI ${ }^{+}$) found $\mathrm{M}^{+} 368.2178, \mathrm{C}_{18} \mathrm{H}_{29} \mathrm{~N}_{3} \mathrm{O}_{5}$ requires $\mathrm{M}^{+} 368.2180 .{ }^{1} \mathrm{H}$ NMR (600 MHz, Deuterium oxide) $\delta 9.20(\mathrm{~s}, 1 \mathrm{H}), 7.02-6.97(\mathrm{~d}, \mathrm{~J}=4.1 \mathrm{~Hz}, 1 \mathrm{H}), 6.24$ $(\mathrm{d}, J=4.1 \mathrm{~Hz}, 1 \mathrm{H}), 4.55(\mathrm{~s}, 2 \mathrm{H}), 4.16(\mathrm{~m}, 2 \mathrm{H}), 4.00(\mathrm{~d}, \mathrm{~J}=6.0 \mathrm{~Hz}, 1 \mathrm{H}), 3.68(\mathrm{t}, \mathrm{J}=6.7$ $\mathrm{Hz}, 1 \mathrm{H}), 3.57(\mathrm{~s}, 1 \mathrm{H}), 1.66(\mathrm{~m}, 2 \mathrm{H}), 1.62(\mathrm{~m}, 2 \mathrm{H}), 1.28(\mathrm{~m}, 2 \mathrm{H}), 0.81(\mathrm{~s}, 2 \mathrm{H}), 0.80(\mathrm{~s}$, 3H), 0.78(s, 3H) (Figure S2). IR (neat) v 3360, 2970, 2930, 2870, 1640, 1590, 1520 , $1450,1410,1330,1160,1020,785 \mathrm{~cm}^{-1}$.

BOC-TBS-Pyrr-Ala-(2): HRMS (ESI ${ }^{+}$) found $[\mathrm{M}+\mathrm{Na}]^{+}$562.2932, $\mathrm{C}_{26} \mathrm{H}_{45} \mathrm{~N}_{3} \mathrm{O}_{7} \mathrm{SiNa}$ requires $\mathrm{M}^{+}$562.2919. ${ }^{1} \mathrm{H} \mathrm{NMR}(600 \mathrm{MHz}$, Methanol-d4) $\delta 9.31(\mathrm{~s}, 1 \mathrm{H}), 6.87(\mathrm{~d}, \mathrm{~J}=$ $4.0 \mathrm{~Hz}, 1 \mathrm{H}), 6.13(\mathrm{~d}, \mathrm{~J}=4.0 \mathrm{~Hz}, 1 \mathrm{H}), 4.65(\mathrm{~s}, 2 \mathrm{H}), 3.64-3.62(\mathrm{~m}, 4 \mathrm{H}), 1.82-1.74(\mathrm{~m}$, 4H), $1.35(\mathrm{~s}, 12 \mathrm{H}), 0.81(\mathrm{~s}, 9 \mathrm{H}), 0.00(\mathrm{~s}, 6 \mathrm{H})$ (Figure S3). IR (neat) v 3342, 2950, 2934 , $2856,1699,1656,1461,1365,1253,1168,1070,837,780 \mathrm{~cm}^{-1}$.

Pyrr-Ala-(4): HRMS $\left(\mathrm{ESI}^{+}\right)$found $[\mathrm{M}+\mathrm{H}]^{+}$326.1721, $\mathrm{C}_{15} \mathrm{H}_{23} \mathrm{~N}_{3} \mathrm{O}_{5}$ requires $\mathrm{M}^{+}$ 326.1710. ${ }^{1} \mathrm{H}$ NMR (600 MHz, Deuterium oxide) $\delta 9.25$ (s, 1H), 7.04 (d, J=4.1 Hz, $1 \mathrm{H}), 6.28(\mathrm{~d}, \mathrm{~J}=4.1 \mathrm{~Hz}, 1 \mathrm{H}), 4.59(\mathrm{~s}, 2 \mathrm{H}), 4.25-4.15(\mathrm{~m}, 2 \mathrm{H}), 4.11-3.96(\mathrm{~m}, 1 \mathrm{H}), 3.85$ $(\mathrm{t}, \mathrm{J}=6.7 \mathrm{~Hz}, 1 \mathrm{H}), 1.87-1.73(\mathrm{~m}, 2 \mathrm{H}), 1.73-1.58(\mathrm{~m}, 2 \mathrm{H}), 1.37-1.29(\mathrm{~m}, 2 \mathrm{H}), 1.26(\mathrm{~d}, \mathrm{~J}$ $=7.3 \mathrm{~Hz}, 3 \mathrm{H})$ (Figure S4). IR (neat) v 3360, 2940, 2860, 1660, 1450, 1410, 1150, 783 $\mathrm{cm}^{-1}$. 


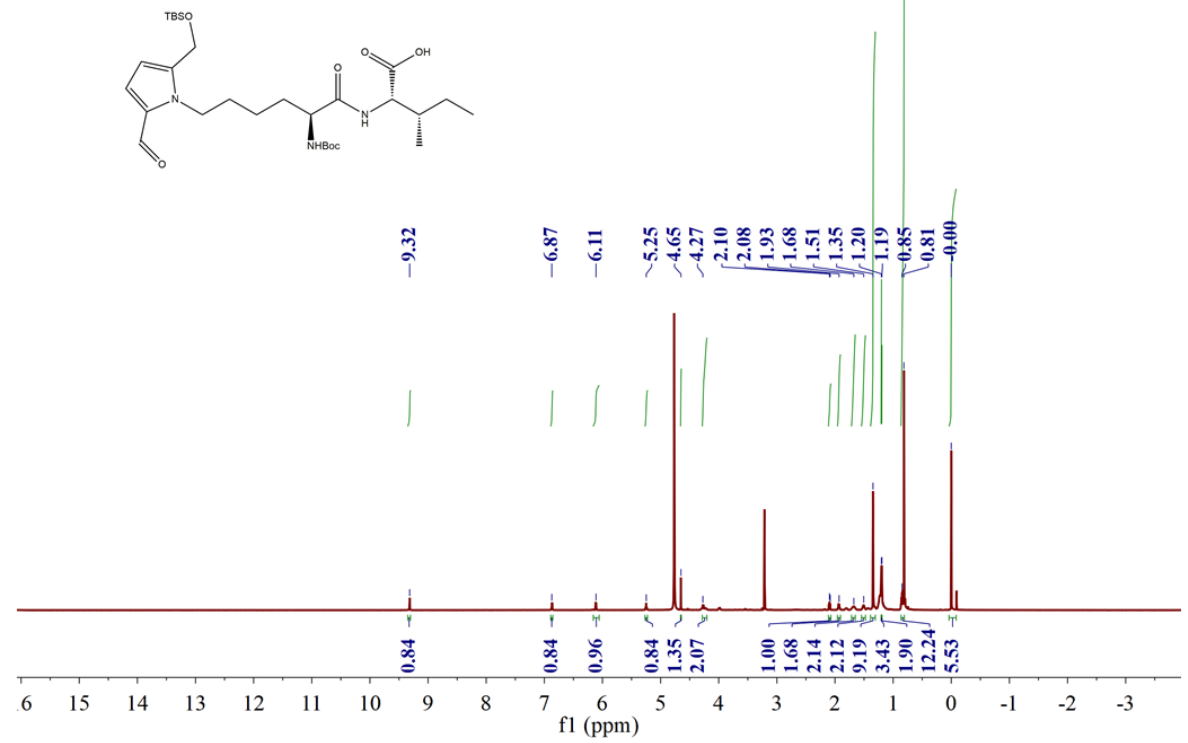

Figure S1 ${ }^{1} \mathrm{H}$ NMR spectrum of Boc-TBS-Pyrr-Ile

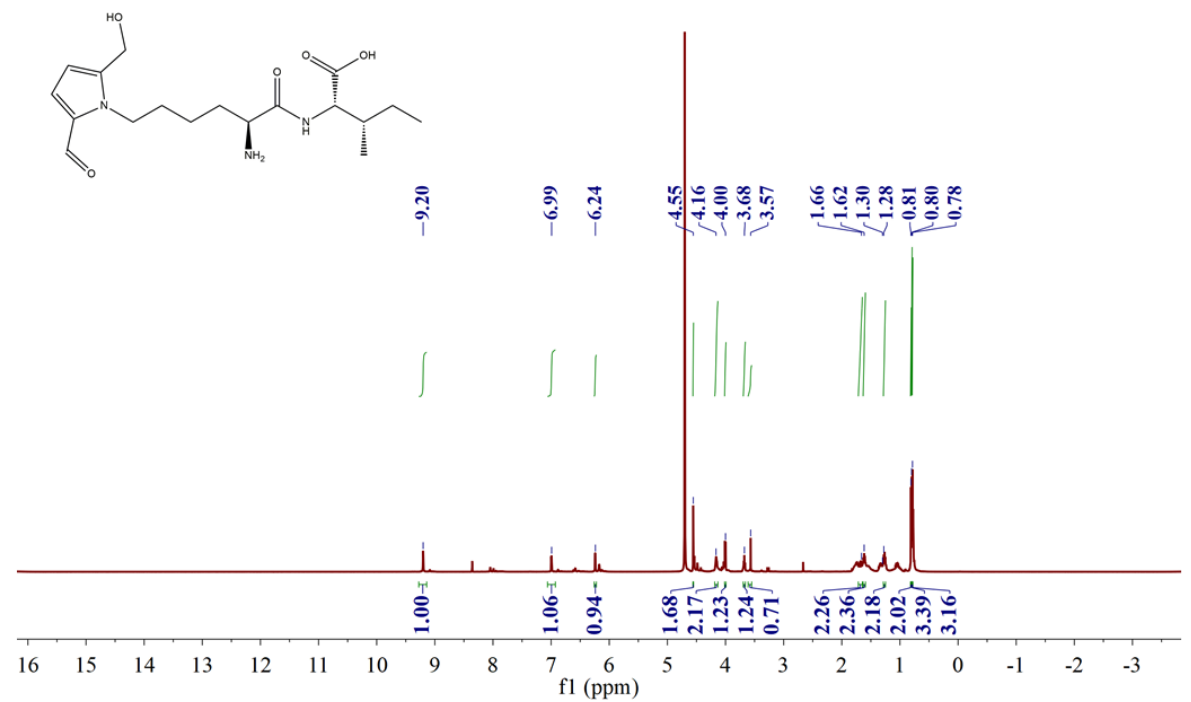

Figure S2 ${ }^{1} \mathrm{H}$ NMR spectrum of Pyrr-Ile 


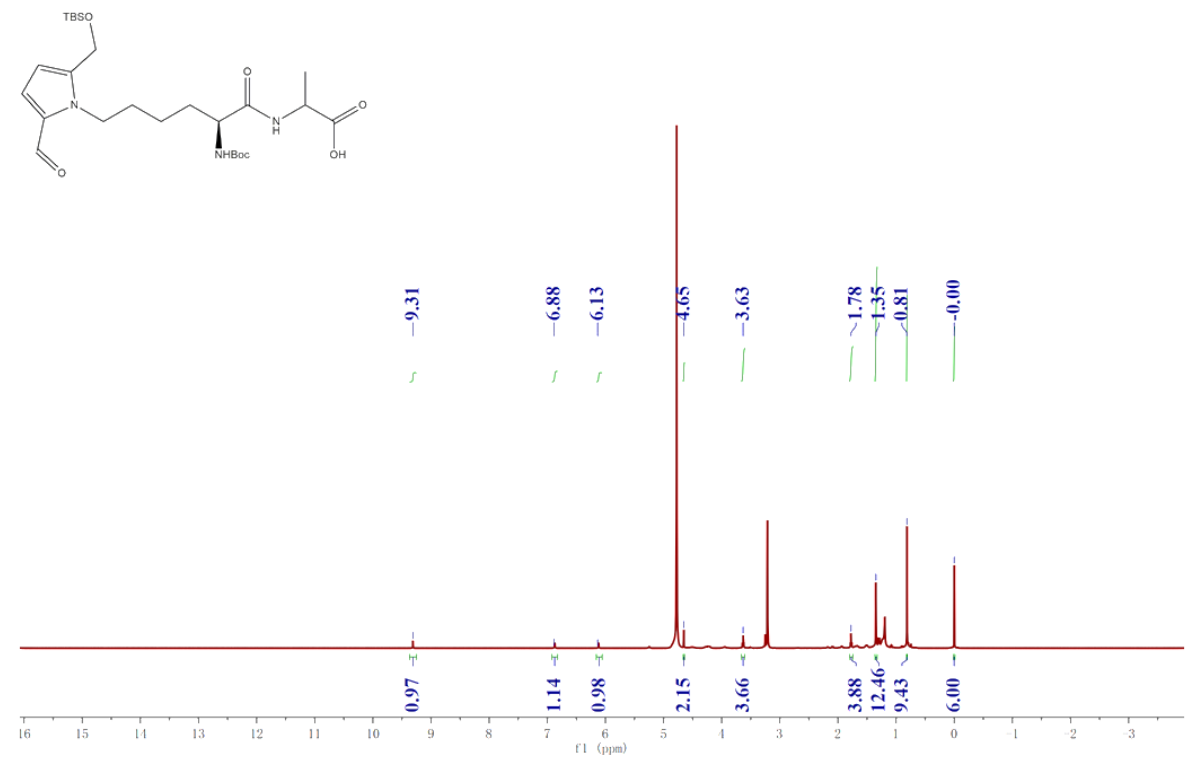

Figure S3 ${ }^{1} \mathrm{H}$ NMR spectrum of Boc-TBS-Pyrr-Ala
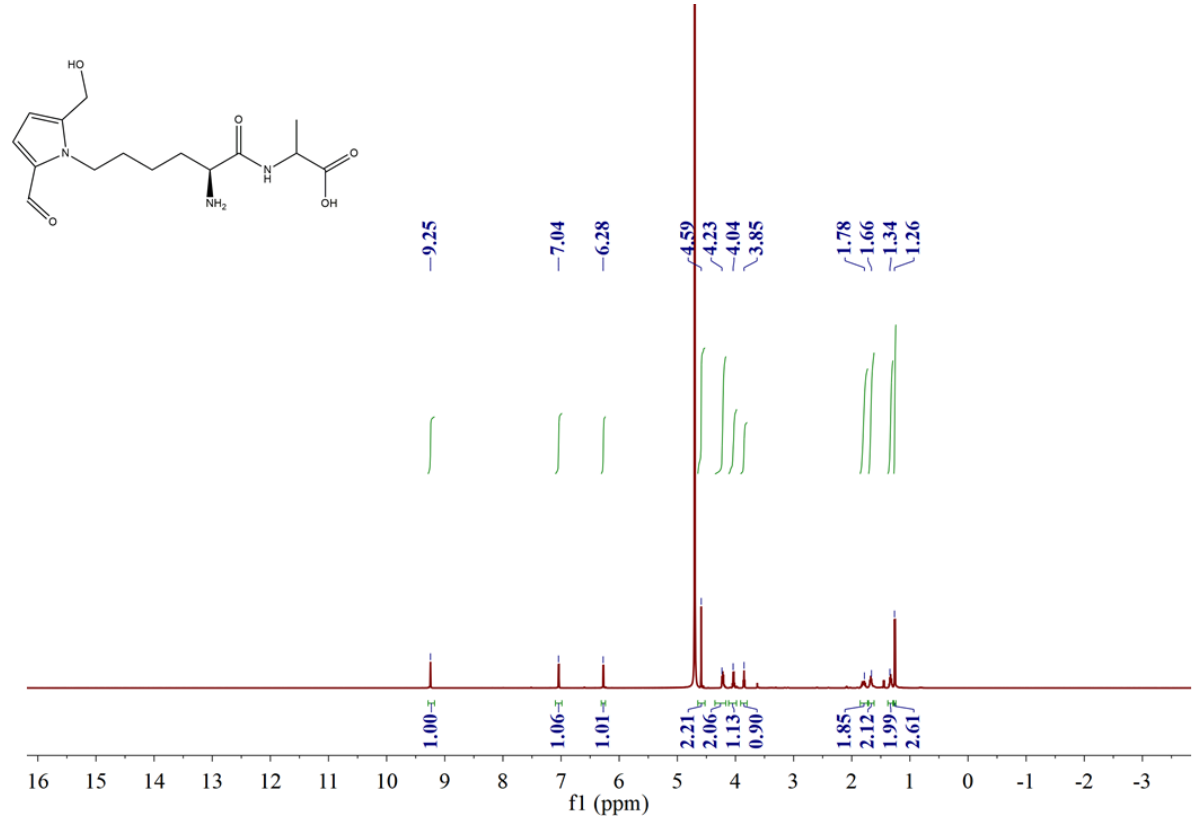

Figure S4 ${ }^{1} \mathrm{H}$ NMR spectrum of Pyrr- Ala 


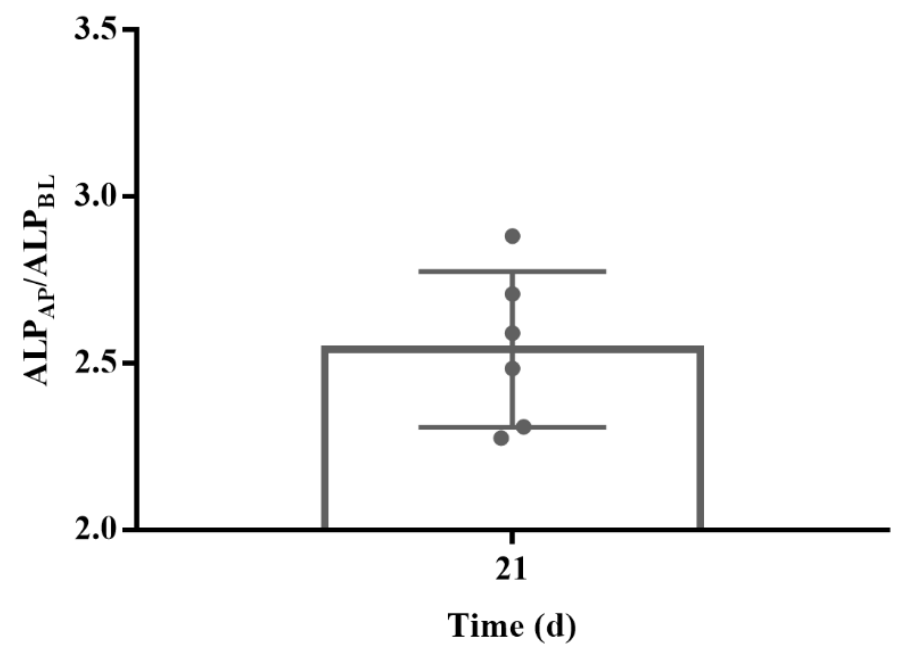

Figure S5 Ratio of alkaline phosphatase activity between apical side (AP) to basolateral side (BL) of Caco-2 cell monolayers ( $A L P_{A P} / A L P B L$, values are mean $\pm S D, n=6$ ). 


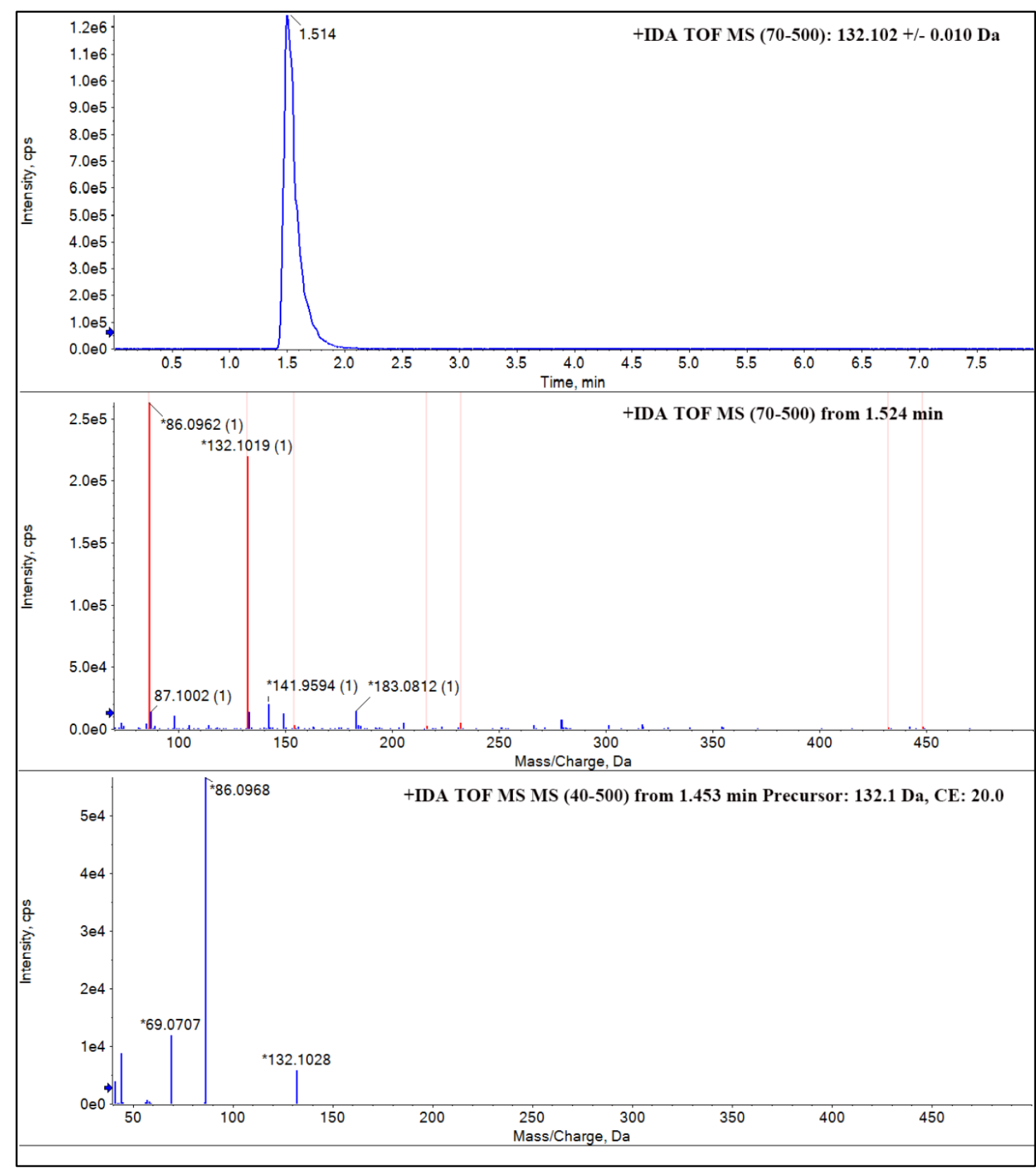

Figure S6 HRMS of isoleucine standard 


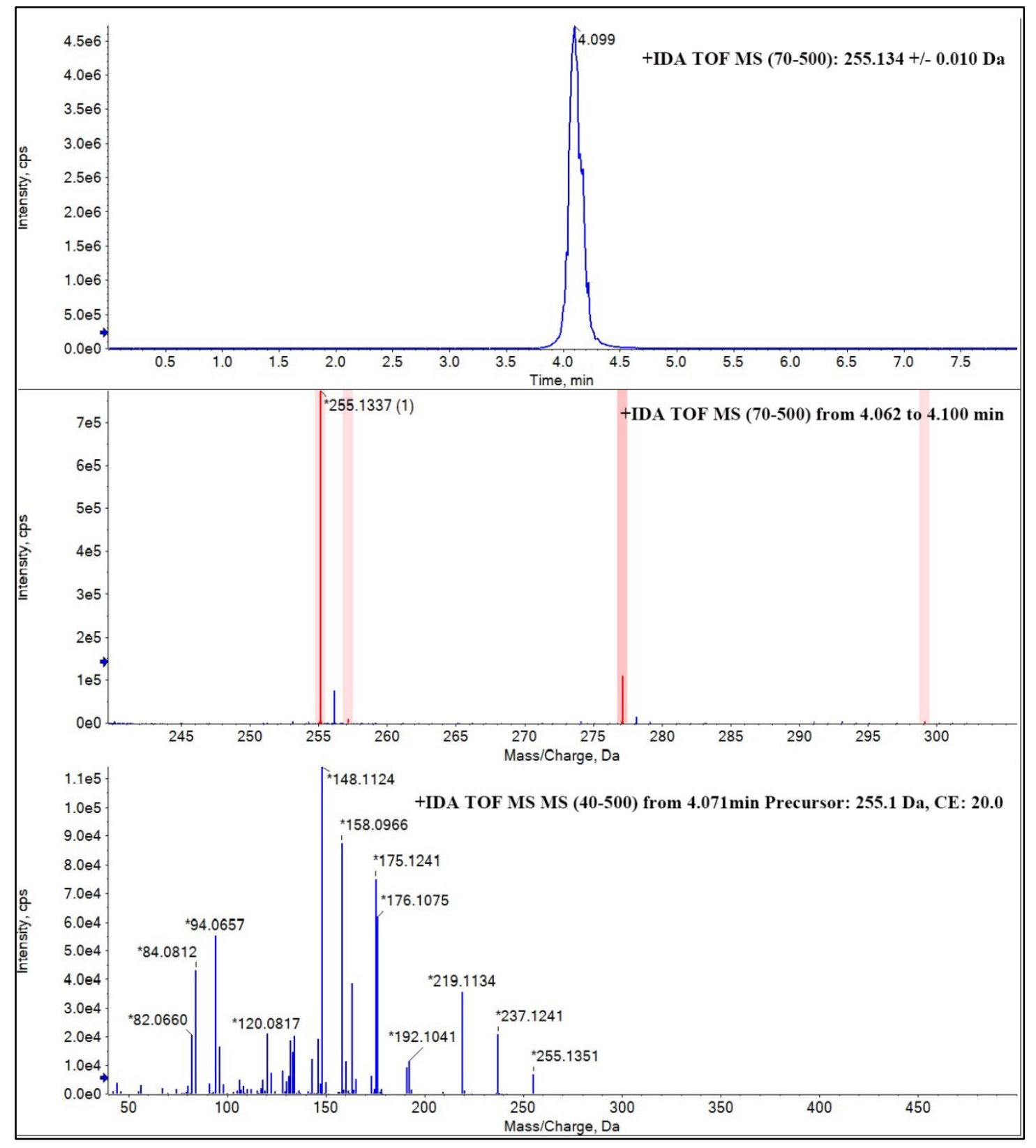

Figure S7 HRMS of free pyrraline standard 


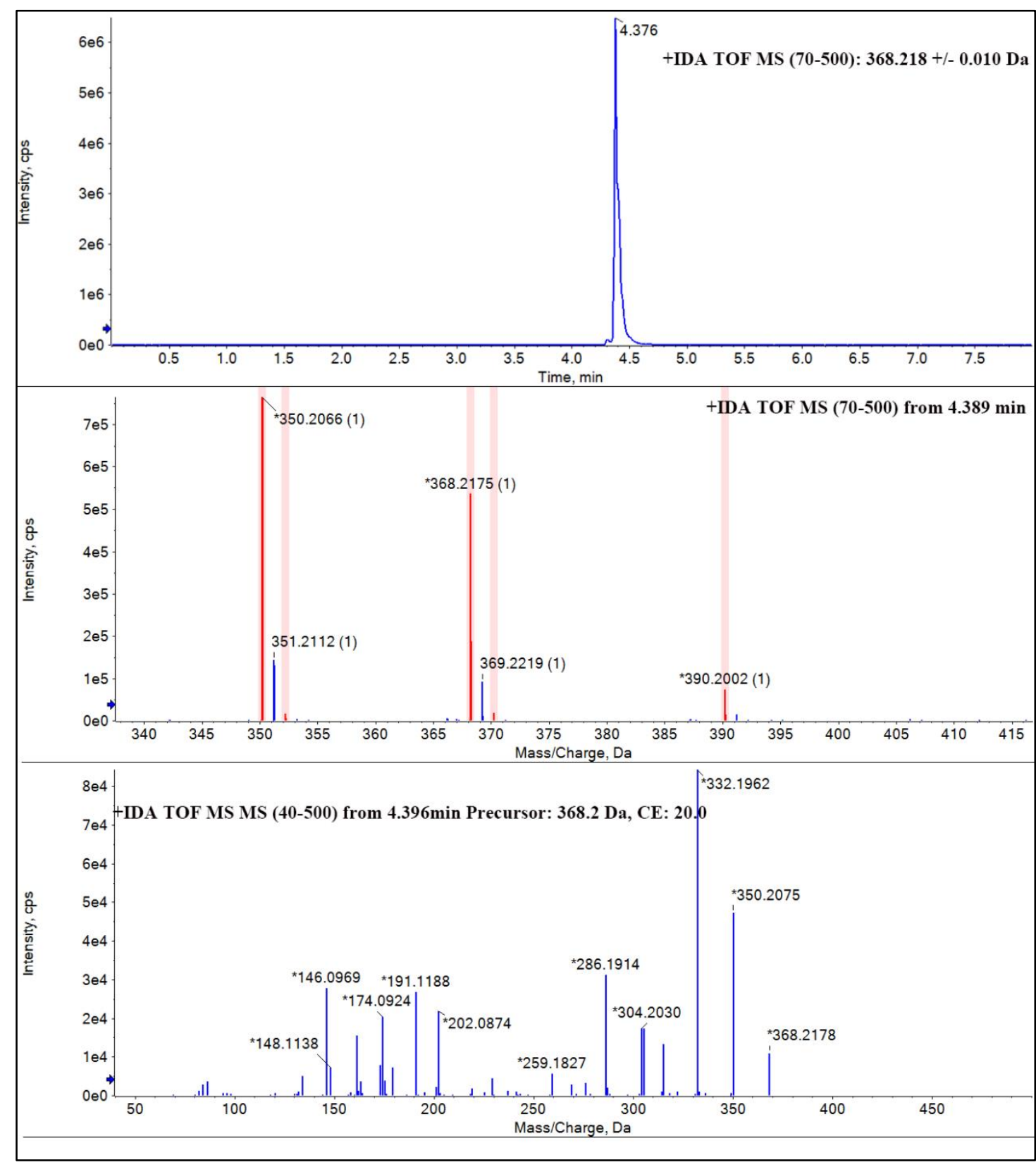

Figure S8 HRMS of Pyrr-Ile standard 


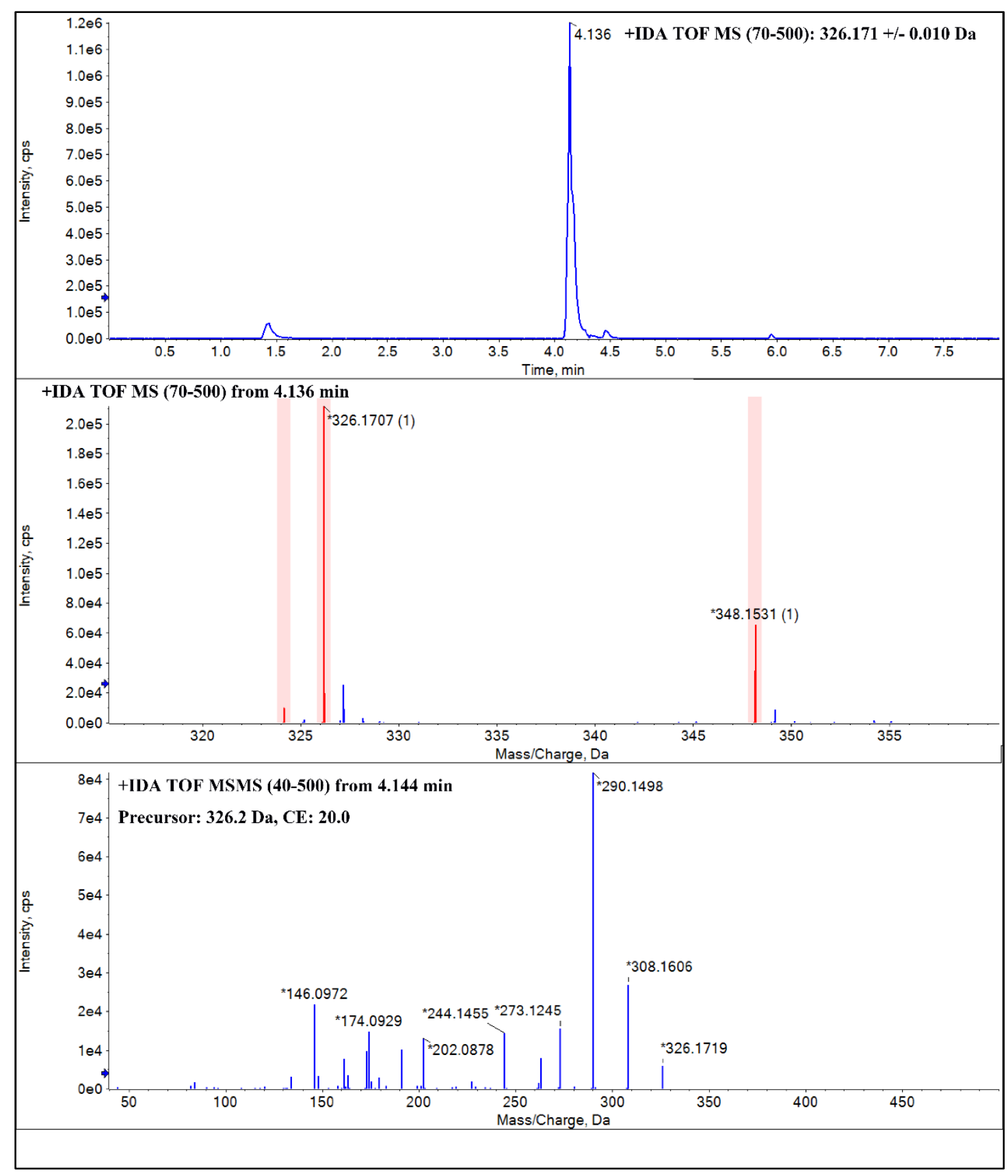

Figure S9 HRMS of Pyrr-Ala standard

\section{References}

1. Li, J.; Chen, L.; Wu, W.; Zhang, W.; Ma, Z.; Cheng, Y.; Du, L.; Li, M., Discovery of Bioluminogenic Probes for Aminopeptidase N Imaging. Analytical Chemistry 2014, 86 (5), 2747-2751. 